\title{
Florística e estrutura de comunidades arbóreas em cerrados de Rondônia, Brasil
}

\author{
Izildinha Souza MIRANDA ${ }^{1}$, Samuel Soares ALMEIDA², Paulo Jorge DANTAS ${ }^{\dagger}$
}

\begin{abstract}
RESUMO
O cerrado constitui o segundo maior e mais diverso bioma brasileiro, configurando um heterogêneo mosaico fisionômico e florístico. Mais de seis mil espécies de fanerógamas são reportadas para o cerrado do Brasil Central. Este trabalho teve o objetivo de descrever e analisar a flora e a estrutura de quatro comunidades de árvores dos cerrados sensu latu do município de Vilhena, Rondônia, Brasil. As áreas estudadas foram: cerradão (Área 1), campo sujo (Área 2) e duas comunidades do cerrado sensu stricto (Áreas 3 e 4). Em cada área foi posicionado aleatoriamente um transecto de $10 \times 1000 \mathrm{~m}$ (1 ha), dividido em 100 parcelas de $10 \mathrm{X} 10 \mathrm{~m}$. Todas as plantas vivas e mortas com circunferência do tronco e" $10 \mathrm{~cm}$ ao nível do solo foram registradas. O cerradão apresentou a maior riqueza específica, estrutura de tamanho e área basal. As percentagens de árvores mortas foram mais altas nas áreas de cerradão e campo sujo, com ocorrência de fogo recente. As famílias mais importantes foram Vochysiaceae, Ochnaceae, Clusiaceae, Malpighiaceae e Melastomataceae. As espécies mais importantes foram Qualea multiflora, Byrsonima crassifolia (área 1), Eugenia sp., Miconiasp. 1 (área 2), Ouratea hexasperma, Caraipa savannarum (área 3), Ouratea hexasperma, Pouteria sp.3 (área 4). A maioria das espécies ocorre somente em uma área, sugerindo que a priorização de áreas para conservação deve envolver todo o mosaico de cerrados existentes. A mortalidade associada ao fogo é diferenciada entre as áreas, talvez induzida pelo grau de flamabilidade, recorrência do fogo e de fatores históricos do uso da terra.
\end{abstract}

PALAVRAS-CHAVE

Amazônia, Árvore, Fitossociologia, Flora.

\section{Flora and structure of tree communities in dry savannas in Rodônia, Brazil}

\section{ABSTRACT}

The Neotropical savannas, locally called "cerrado", are Brazil's second largest and diverse biome, constituting a heterogeneous physiognomic and floristic mosaic from which more than 6,000 species have been reported to date. This study describes and analyzes the floras and structures offour tree communities from dry savannas in the municipality of Vilhena, Rondônia, Brazil. The study areas were: savanna with trees "cerradão" (Area 1), savannas wich bushes or "campo sujo" (Area 2), and two savanna woodlands or "cerrado" sensu stricto (Areas 3 and 4). In each area an transect measuring $10 \times 1,000 \mathrm{~m}(1 \mathrm{ha})$ was placed at random and divided into 100 quadrats of $10 \times 10 \mathrm{~m}$. All living and dead woody plants with circumference ${ }^{3} 10 \mathrm{~cm}$ at ground level were included in the survey. "cerradão" was the richest in species, had the greatest plant diversity, and showed the largest basal area. The percentages of dead trees were highest in areas that had been recently burned. The most species-rich and abundant plant families were Vochysiaceae, Ochnaceae, Guttiferae, Malpighiaceae and Melastomataceae. The most important species were Qualea multiflora and Byrsonima crassifolia (area 1), Eugenia sp. and Miconia sp. 1. (area 2), Ouratea hexasperma and Caraipa savannarum (area 3) Ouratea hexasperma and Pouteria sp3. (area 4). Most species (53\%; $N=92$ spp.) were found in only one of the four areas, indicating that the selection of priority areas for biological conservation should take into account the whole of the savanna mosaic. Tree mortality caused by fire was different among the four study areas, probably as a function of burning recurrency, the degree of flammability, and historical factors involving land use.

\section{KEYWORDS}

Amazonian, Flora, Phytosociology, Tree.

\footnotetext{
${ }^{1}$ Universidade Federal Rural da Amazônia, Departamento de Ciências Florestais, Av. Pres.Tancredo Neves n. ${ }^{2}$ 2501, Caixa Postal 917, CEP66.077-530, Belém, PA, Brasil. izildinhamiranda@uol.com.br ${ }^{2}$ Museu Paraense Emílio Goeldi, Departamento de Botânica, Av. Magalhães Barata, 376, C.P. 399, CEP 66040-170, Belém, PA, Brazil. salmeida@museu-goeldi.br $\dagger$ In memorian
} 


\section{INTRODUÇÃO}

A paisagem dos cerrados brasileiros é composta por um mosaico fito-fisionômico e florístico, caracterizada por vegetação savânica aberta, xeromórfica e rica em espécies (Huber, 1987), especialmente aquelas com distribuição restrita e endêmica. Esse bioma é o segundo em extensão, com cerca de dois milhōes de $\mathrm{km}^{2}$, correspondendo a $23 \%$ do território brasileiro.

No passado, a dinâmica geo-climática foi determinante para a origem e diversificação dos cerrados. Atualmente, as variáveis edáficas e a sazonalidade climática são importantes para explicar a heterogeneidade ambiental verificada nesse bioma. Entre as propriedades dos solos apontadas como relevantes, pode-se citar o teor do alumínio, o teor das bases catiônicas trocáveis e dos microelementos, além de propriedades físicas como a textura, a capacidade de armazenamento de água, a profundidade, entre outras (Eiten, 1972; Goodland \& Pollard, 1973; Montgomery \& Askew, 1983; Ratter \& Dargie, 1992). O regime pluviométrico também é fundamental, especialmente, o total e a distribuição anual das chuvas, assim como o período da estação seca (Huber, 1982; Oliveira Filho e Martins, 1986; Furley \& Ratter, 1988; Oliveira Filho et. al., 1989). Outros fatores como fogo, herbivoria e perturbaçoes antrópicas também desempenham papéis diferenciados e importantes em cada região, sendo considerados como determinantes secundários desses ecossistemas (Gibbs et al., 1983; Medina, 1987; Borhidi, 1988; Pagano et al., 1989; Durigan et al., 1994; Hoffmann, 1996).

Os cerrados do Brasil Central e mesmo aqueles da transição para a Amazônia, como os de Rondônia, estão situados em zonas de forte estacionalidade, onde o fogo é um evento freqüente que influencia, inclusive, a dinâmica da vegetação. A origem do fogo pode ser tanto natural ou fortuita devido ao grau de ignição, como acidental, de origem antrópica, quando utilizado na agricultura para limpeza de pastos, de áreas de culturas ou mesmo fogo induzido ou intencional (Raw \& Hay, 1985).

O grau de ignição ou o potencial de flamabilidade, é dependente de diversos fatores locais, físicos e mesmo históricos, tais como: déficit hídrico, duração do período seco, estrutura da vegetação e grau de intermitência do evento. A combinação desses fatores faz com que cada área ou zona apresente susceptibilidade diferenciada ao fogo.

Os efeitos e os mecanismos ecofisiológicos e morfológicos das plantas de cerrado, e por extensão as de savanas, para resistirem ou conviverem com o fogo são bem documentados na literatura científica (Coutinho, 1978; Raw \& Hay, 1985; Hoffman, 1996; Oliveira Filho et al., 1989).

A flora fanerogâmica nativa do bioma cerrado no Brasil Central, listada por Mendonça et al. (1998), inclui 6.060 espécies e 425 variedades distribuídas em 1.093 gêneros e 151 famílias botânicas, abrangendo todas as formas de vida vegetal e as várias feiçōes de cerrado sensu lato, inclusive os ambientes associados como florestas de galeria, brejos e campos de altitude.

Em Rondônia, os cerrados localizam-se em grandes áreas contínuas, localizadas no sul do estado, na transição entre os domínios da floresta amazônica e dos cerrados do Brasil Central. $\mathrm{Na}$ transição com o bioma amazônico, os cerrados se entrelaçam com florestas abertas com palmeiras e florestas estacionais semideciduais. Compondo ainda esse cenário de "tensão ecológica", existem também, no norte do estado, áreas isoladas ou "ilhas" de cerrado, também conhecidas como savanas amazônicas. Esses fragmentos parecem que foram isolados pela expansão da floresta tropical neste último período úmido interglacial.

No município de Vilhena, ao sul de Rondônia, onde o presente estudo foi realizado, a vegetação de cerrado tem sido continuamente transformada em áreas agrícolas com alto potencial de produção de grãos em grande escala, especialmente soja. O avanço da soja se dá tanto sobre as áreas agrícolas e pastos abandonados, como sobre os remanescentes de cerrados. $\mathrm{O}$ processo de dizimação dos cerrados neste estado é semelhante ao ocorrido no Centro Oeste, onde grandes áreas foram convertidas em plantios homogêneos de soja, graças às novas tecnologias de correção do solo, seleção de sementes e mecanização agrícola.

Apesar da existência de muitos estudos sobre a estrutura, dinâmica e composição dos vários grupos biológicos nos cerrados brasileiros, o conhecimento sobre a flora e a estrutura da vegetação de cerrados, nas regiōes de transição entre os dois principais biomas brasileiros, é praticamente inexistente. Dessa forma, há uma grande necessidade de estudos sobre a flora e estrutura desse ambiente, não somente para aumentar o conhecimento científico sobre o mesmo, como também para subsidiar a escolha de áreas prioritárias para conservação de tratos representativos da variação ambiental e da biodiversidade do bioma cerrado, na junção com a floresta amazônica.

Este trabalho tem como objetivos descrever e analisar a flora e estrutura de quatro comunidades de árvores de cerrados do município de Vilhena, em Rondônia, visando aumentar o conhecimento sobre a composição florística e o porte desses ambientes na Amazônia, e, ainda, subsidiar a priorização de áreas para conservação.

\section{MÉTODOS}

\section{ÁREAS DE ESTUDO}

As áreas de cerrado estudadas estão localizadas no município de Vilhena, sul do estado de Rondônia, já na divisa com o Estado do Mato Grosso, entre as coordenadas 12¹7'50" - 12³2'6,3" de latitude Sul e 60'12'32,8"- 60'25'10,7"de longitude Oeste, com altitudes que variam entre 347 a $565 \mathrm{~m}$ (Tabela 1). Essas áreas estão entre os rios Roosevelt e Comemoração, formadores da bacia do rio Madeira e drenam para o Norte. Tais áreas estão localizadas numa zona de importância biogeográfica da Chapada 
Tabela 1 - Localização das áreas estudadas e suas fisionomias de acordo com Ribeiro \& Walter (1998). Município de Vilhena, Rondônia.

\begin{tabular}{|c|c|c|c|c|}
\hline Área & $\begin{array}{c}\text { Fisionomia da } \\
\text { Vegetação }\end{array}$ & Latitude-S & Longitude-W & Altitude (m) \\
\hline Área 1 & $\begin{array}{l}\text { Cerradão (transição } \\
\text { para cerrado sensu } \\
\text { stricto) }\end{array}$ & $12^{0} 17^{\prime} 50.0$ " & $60^{\circ} 24$ ' 31.2 " & 347 \\
\hline Área 2 & Campo sujo & $12^{\circ} 31^{\prime} 26.5$ " & $60^{\circ} 12$ ' 32.8 " & 565 \\
\hline Área 3 & $\begin{array}{l}\text { Cerrado sensu } \\
\quad \text { stricto }\end{array}$ & $12^{\circ} 28$ ' 41.0 " & $60^{\circ} 25^{\prime} 10.7$ " & 520 \\
\hline Área 4 & $\begin{array}{l}\text { Cerrado sensu } \\
\text { stricto }\end{array}$ & $12^{\circ} 32^{\prime} 06.3$ " & $60^{\circ} 23$ ' 46.5 " & 432 \\
\hline
\end{tabular}

dos Parecis, uma vez que para Leste drenam os rios formadores da bacia do Tapajós, constituindo-se então num divisor de águas.

As áreas de estudo foram selecionadas levando em consideração a representatividade dos tipos de cerrado na regiáo de Vilhena e a facilidade de acesso. A terminologia utilizada para os cerrados seguiu aquela apresentada e discutida por Ribeiro \& Walter (1998).

O clima das áreas éo tropical úmido, quente, com sazonalidade marcante, caracterizado por estação seca muito bem definida. As temperaturas médias anuais estão entre 22 e $24^{\circ} \mathrm{C}$ e as precipitaçôes pluviométricas médias anuais entre 2000 e 2250 $\mathrm{mm}$ e umidade relativa média do ar em torno de 70 e $80 \%$ (Nimer, 1991).

Os solos predominantes são latossolos arenosos, bem drenados, ácidos, com altas concentrações de alumínio, baixa concentração de matéria orgânica e de bases trocáveis como cálcio, magnésio, potássio e fósforo (Tabela 2). Os níveis de micronutrientes como ferro, cobre e zinco são satisfatórios. Os valores estão dentro daqueles reconhecidos para os solos de cerrados no Brasil Central e de savanas neotropicais em geral (Montgomery \& Askew, 1983).

Tabela 2 - Características químicas dos solos superficiais $(0-20 \mathrm{~cm})$ de quatro áreas de cerrado. Município de Vilhena, Rondônia.

\begin{tabular}{ccccc}
\hline & Área 1 & Área 2 & Área 3 & Área 4 \\
\hline Areia(\%) & 86 & 86 & 87 & 87 \\
\hline Argila (\%) & 11 & 11 & 10 & 10 \\
\hline Silte (\%) & 3 & 3 & 3 & 3 \\
\hline $\mathrm{pH} \mathrm{H} \mathrm{O}_{2}$ & 4,3 & 4,6 & 4,7 & 4,7 \\
\hline $\mathrm{pH} \mathrm{K} \mathrm{Cl}$ & 3,7 & 4,0 & 4,0 & 4,1 \\
\hline $\mathrm{Matéria} \mathrm{orgânica}$ & 2,6 & 0,9 & 0,7 & 1,0 \\
\hline $\mathrm{Al}$ (meq/100g) & 1,38 & 0,61 & 0,46 & 0,31 \\
\hline $\mathrm{Ca}(\mathrm{meq} / 100 \mathrm{~g})$ & 0,18 & 0,24 & 0,16 & 0,18 \\
\hline $\mathrm{Mg}$ (meq/100g) & 0,07 & 0,10 & 0,03 & 0,07 \\
\hline $\mathrm{K}$ (meq/100g) & 0,04 & 0,01 & 0,01 & 0,01 \\
\hline $\mathrm{P} \mu \mathrm{g} / \mathrm{g}$ & 2,8 & 1,5 & 0,7 & 0,2 \\
\hline $\mathrm{Fe} \mu \mathrm{g} / \mathrm{g}$ & 118,3 & 65,1 & 94,1 & 59,8 \\
\hline $\mathrm{Cu}$ & 0,3 & 0,1 & 0,1 & 0,1 \\
\hline
\end{tabular}

\section{MÉTODOS DE CAMPO E DE ANÁLISES}

Em cada área selecionada foi aleatoriamente posicionado um transecto de $10 \times 1.000 \mathrm{~m}$, totalizando $10.000 \mathrm{~m}^{2}$ ou 1 hectare. O transecto foi dividido em 100 parcelas de $10 \times 10 \mathrm{~m}$. Dentro de cada parcela foram inventariadas todas as plantas, vivas e mortas, em pé com circunferência e" $10 \mathrm{~cm}$ ao nível do solo. Além da medida da circunferência, foi medida a altura total, coletado material botânico e realizada uma pré-identificação em campo.

O material botânico foi identificado com auxílio de literatura taxonômica e por comparação com material de herbário. Parte das espécies foi apenas morfotipada, porque o material botânico coletado estava estéril. As exsicatas contendo material fértil foram depositadas no herbário “João Murça Pires”, do Museu Paraense Emílio Goeldi.

A análise florística incluiu a densidade de indivíduos, números de espécies, gêneros e famílias botânicas registradas por área, além da indicação dos gêneros e famílias mais ricos em espécies. A análise da estrutura incluiu a diversidade florística, calculada através do índice de Shannon-Wiener $\left(\mathrm{H}^{\prime}\right)$ baseado em logaritmos naturais; a equabilidade (E) foi calculada usando o índice de Pielou de acordo com Magurran (2004). Também foram incluídas a média das alturas e a totalização da área basal (AB), por área estudada.

Foram analisados parâmetros convencionais de fitossociologia, como abundâncias absoluta $(\mathrm{N})$ e relativa $(\mathrm{NR})$, freqüência relativa (FR), dominância relativa (DoR) e o índice de valor de importância específica (IVI). Todos os parâmetros relativos estão apresentados em percentagem e foram obtidos para cada espécie, por área estudada. Esses parâmetros foram calculados de acordo com Brower et al. (1997).

Para fins de análise, as espécies foram separadas entre aquelas com ocorrência restrita, quando registrada em somente uma área; ocorrência ocasional, quando detectada em duas áreas e com ocorrência irrestrita ou ampla, quando foi inventariada em três ou quatro áreas.

\section{RESULTADOS}

Os transectos inventariados nas quatro áreas incluíram 5.215 troncos, sendo 4.828 (92,58 \%) pertencentes a árvores vivas e $387(7,42 \%)$ a árvores mortas. Esses indivíduos pertencem a 92 espécies ou morfoespécies, 61 gêneros e 38 famílias botânicas (Anexo I).

O transecto inventariado no cerradão (Área 1) apresentou total de 1.609 indivíduos, sendo que 211 estavam mortos $(13,11$ $\%)$. A flórula de árvores vivas incluiu 60 espécies pertencentes a 46 gêneros e 28 famílias botânicas. A área basal por hectare atingiu $12,44 \mathrm{~m}^{2}$ (Tabela 3). As famílias Vochysiaceae (4 espécies), Fabaceae (4 espécies), Malpighiaceae (4 espécies) e Rubiaceae (5 espécies) foram as mais ricas e abundantes nessa 
área. Os gêneros melhor representados foram Byrsonima com quatro espécies; Qualea e Eriotheca com três espécies; Davilla, Miconia, Erythroxylum, Pouteria, Kielmeyera e Eugenia com duas espécies cada (Anexo I).

O cerrado tipo campo sujo (Área 2) apresentou 442 indivíduos por hectare, com 72 troncos mortos (16,29\%). O restante dos indivíduos pertencia a 26 espécies, 24 gêneros e 20 famílias (Tabela 3). Aárea basal desse tipo de cerrado foi a menor, registrando-se somente $1,72 \mathrm{~m}^{2}$ por hectare (Tabela 3). As famílias mais abundantes no campo sujo foram Myrtaceae (1 espécie), Melastomataceae (3 espécies) e Sapotaceae (1 espécie). Os gêneros Miconia e Byrsonima apresentaram duas espécies cada (Anexo I).

No cerrado sensu stricto (Área 3) foram inventariados 1.353 indivíduos, com 60 troncos mortos $(4,43 \%)$. Os demais pertenciam a 39 espécies, 33 gêneros e 24 famílias. A área basal obtida foi de $8,71 \mathrm{~m}^{2}$ (Tabela 3). As famílias Ochnaceae (1 espécie), Clusiaceae (2 espécies), Myrtaceae (3 espécies), Vochysiaceae (3 espécies) e Melastomataceae (3 espécies) foram as mais abundantes e ricas nesse ambiente. $O$ gênero Byrsonima foi o único que apresentou três espécies; os gêneros Eugenia, Vochysia, Miconia e Erythroxylum apresentaram duas espécies cada um (Anexo I).

O segundo cerrado sensu stricto (Área 4) incluiu 1.811 indivíduos, sendo que 44 (2,43\%) estavam mortos. Os demais indivíduos vivos estavam distribuídos em 45 espécies, 38 gêneros e 30 famílias. A área basal calculada ficou em $6,63 \mathrm{~m}^{2}$ por hectare (Tabela 3). As famílias mais importantes foram Ochnaceae, Vochysiaceae, Melastomataceae, Myrtaceae e Sapotaceae (Anexo I). Essa área apresentou florística mais rica e estrutura de menor porte quando comparada com a área anterior.

Em termos florísticos, a área de cerradão juntamente com as áreas de cerrado sensu stricto, apresentaram flórulas mais ricas em espécies, gêneros e famílias.

As distribuições de circunferências das quatro áreas revelaram padrão decrescente, com muitos indivíduos nas classes de tamanho menores (Figura 1). O mesmo padrão de distribuição

Tabela 3 - Estrutura de quatro comunidades arbóreas de cerrado. Município de Vilhena, Rondônia.

\begin{tabular}{ccccc}
\hline Parâmetros & Área 1 & Área 2 & Área 3 & Área 4 \\
\hline No. Espécies $(\mathrm{N}=92)$ & 60 & 26 & 39 & 45 \\
\hline No. Gêneros $(\mathrm{N}=61)$ & 46 & 24 & 33 & 38 \\
\hline No. Famílias $(\mathrm{N}=38)$ & 28 & 20 & 24 & 30 \\
\hline $\begin{array}{c}\text { Altura média }(\mathrm{m})+ \\
\text { desvio padrão }\end{array}$ & $3.72 \pm 2.43$ & $1.69 \pm 0.96$ & $2.28 \pm 1.42$ & $2.01 \pm 0.97$ \\
No. Árvores/hectare & 1.609 & 442 & 1.353 & 1.811 \\
Área Basal $(\mathrm{m} 2)$ & 12,44 & 1,72 & 8,71 & 6,63 \\
\hline Diversidade $\left(\mathrm{H}^{\prime}\right)$ & 3,45 & 2,34 & 2,63 & 2,90 \\
\hline Equabilidade $(\mathrm{E})$ & 0,84 & 0,72 & 0,72 & 0,76 \\
\hline \% de Troncos Mortos/ha & 13,11 & 16,29 & 4,43 & 2,43 \\
\hline
\end{tabular}

foi apresentado pela maioria das principais populaçōes arbóreas das quatro áreas estudadas. A área 4 apresentou a maioria dos indivíduos na classe de circunferência acima de $50 \mathrm{~cm}$, denotando que esse ambiente apresenta estrutura de tamanho de maior porte, apesar de que, em média, sua altura foi inferior ao cerradão (Tabela 3).

A maioria das espécies apresentou ocorrência restrita a uma localidade (49 espécies, 53,26\%). Por sua vez, espécies registradas em três ou quatro áreas totalizam 26 (28,26\%), enquanto aquelas ocasionais, ou com dispersão intermediária que apareceram em 2 áreas, formaram o menor grupo, com 17 espécies (18,48 \% do total) (Tabela 4).

A densidade média das espécies com ocorrência irrestrita (35,70 árvores.ha $\left.{ }^{-1}\right)$ foi mais elevada quando comparada com aquela verificada para as espécies com ocorrência restrita $(24,78$ árvores.ha $\left.{ }^{-1}\right)$ e ocasional (14,94 árvores.ha $\left.{ }^{-1}\right)$ (Tabela 4).

Entre os cerrados estudados, o número de indivíduos mortos na área de cerrado tipo "campo sujo" foi mais elevado, atingindo $16,29 \%$ do total de troncos e $18,60 \%$ da área basal por hectare (Tabela 5). As áreas de cerrado sensu stricto apresentaram os menores valores relativos para densidades $(4,43 \%$ e $2,43 \%$ para as áreas 3 e 4, respectivamente) e áreas basais (4,02 \% e 2,87\% para as áreas 3 e 4 , respectivamente) de troncos mortos (Tabela 5).

\section{ANÁLISE FITOSSOCIOLÓGICA}

As informações sobre os principais parâmetros fitossociológicos calculados para os cerrados estudados em Rondônia estão representadas na Tabela 5. A análise foi concentrada nas cinco espécies de maior IVI, sendo estas relacionadas com as comunidades arbóreas às quais pertenciam.

ÁREA 1: CERRADÃO (TRANSIÇÃO COM CERRADO SENSU STRICTO)

Nesse ambiente, as cinco espécies mais representativas incluíram 30,15\% da abundância relativa total, o que correspondeu a 485 indivíduos.ha ${ }^{-1} ; 36,34 \%$ da dominância relativa da comunidade (Área Basal $=4,51 \mathrm{~m}^{2} \cdot \mathrm{ha}^{-1}$ ) e 26,19\% da freqüência relativa registrada. O IVI expresso pela soma da abundância, dominância com as freqüências relativas, divididas por três, totalizou 30,89 \% nesse grupo de espécies (Tabela 6).

Tabela 4 - Riqueza específica e densidade (média e erro padrão, EP) de acordo com 0 número de áreas de cerrado onde ocorrem as espécies. Município de Vilhena, Rondônia.

\begin{tabular}{ccccccc}
\hline \multirow{2}{*}{ Tipo de Ocorrência } & No. Áreas & No. Spp. & $\%$ & \multicolumn{2}{c}{ Densidade/ha } \\
\cline { 5 - 7 } & & & & & Média & EP \\
\hline Irrestrita & 3 ou 4 & 26 & 28,26 & 35,70 & 12,83 \\
\hline Ocasional & 2 & 17 & 18,48 & 14,94 & 6,96 \\
Restrita ou Exclusiva & 1 & 49 & 53,26 & 24,78 & 5,62 \\
\hline Total ou Média & & 92 & 100,00 & 28,40 & 5,56 \\
\hline
\end{tabular}


Tabela 5 - Abundância (N) e área basal (AB) de troncos vivos e mortos em quatro áreas de cerrado. Município de Vilhena, Rondônia.

\begin{tabular}{cccccccccc}
\hline \multirow{2}{*}{ ÁREA } & \multicolumn{3}{c}{ Troncos Vivos } & \multicolumn{4}{c}{ Troncos Mortos } \\
\cline { 2 - 9 } & $\mathrm{N}$ & $\%$ & $\mathrm{AB}(\mathrm{m} 2)$ & $\%$ & $\mathrm{~N}$ & $\%$ & $\mathrm{AB}(\mathrm{m} 2)$ & $\%$ \\
\hline Cerradão & 1.398 & 86,89 & 11,10 & 89,23 & 211 & 13,11 & 1,34 & 10,77 \\
Campo Sujo & 370 & 83,71 & 1,40 & 81,40 & 72 & 16,29 & 0,32 & 18,60 \\
Cerrado & 1.293 & 95,57 & 8,36 & 95,98 & 60 & 4,43 & 0,35 & 4,02 \\
Cerrado & 1.767 & 97,57 & 6,44 & 97,13 & 44 & 2,43 & 0,19 & 2,87 \\
\hline
\end{tabular}

Qualea multiflora (Vochysiaceae) se destacou nesse grupo, atingindo IVI de 10,39\%. As outras espécies proeminentes nessa comunidade foram Byrsonima crassifolia (Malpighiaceae), $\mathrm{IVI}=6,61$; Kielmeyera rubriflora (Clusiaceae), IVI = 5,37 \%, Byrsonima tomentosa (Malpichiaceae), IVI $=4,29 \% \mathrm{e}$ Sclerolobium sp. (Caesalpiniaceae), IVI = 4,24 (Tabela 6).

\section{ÁREA 2: CAMPO SUJO}

Esse ambiente foi apresentou os maiores valores relativos para os parâmetros fitossociológicos das cinco espécies mais destacadas da comunidade arbórea, em comparação aos demais. Esse grupo de espécies atingiu 64,03 \% da abundância relativa total, correspondente a 283 indivíduos/ha, 60,16\% da dominância obtida pela comunidade (Área Basal = 1,04 m².ha ${ }^{-1}$ ) e 59,31\% da freqüência total. $O$ grupo das cinco espécies mais destacadas na comunidade arbóreo atingiu $61,17 \%$ do total do IVI.

As espécies mais abundantes também foram as mais freqüentes e dominantes, sendo conseqüentemente, as mais importantes na comunidade. Nesse grupo, destacou-se Eugenia sp. (Myrtaceae), IVI $=21,14 \%$; Miconia sp. 1 (Melastomataceae), IVI = 15,01 \%; Pouteria sp.1 (Sapotaceae), IVI = 10,80 \%; Connarus suberosus (Connaraceae), IVI = 8,52\% e Eriotheca sp.1 (Bombacaceae), IVI = 5,70 \% (Tabela 6).

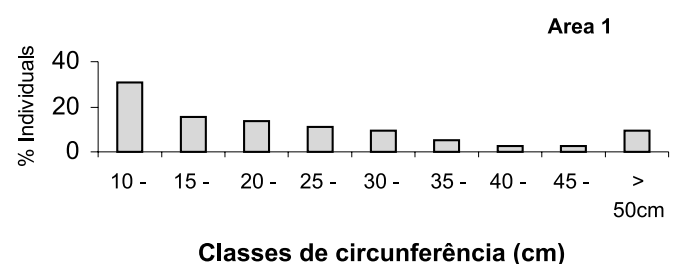

Classes de circunferência (cm)

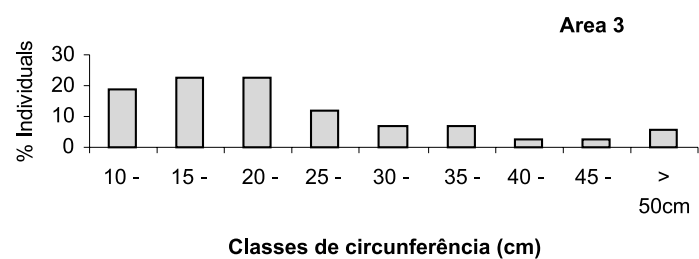

Classes de circunferência $(\mathbf{c m})$

\section{ÁREA 3: CERRADO SENSU STRICTO}

As cinco espécies mais representativas, nessa área, apresentaram valores relativos dos parâmetros fitossociológicos ligeiramente maiores quando comparada com outra área de cerrado sensu stricto (Área 4). A abundância relativa alcançou 60,98 \%, correspondendo a 825 indivíduos.ha ${ }^{-1}$. A dominância relativa para esse grupo foi de $59,56 \%\left(\mathrm{AB}=5,19 \mathrm{~m}^{2} \cdot \mathrm{ha}^{-1}\right)$, enquanto que a frequência relativa atingiu valor inferior aos demais, com $41,86 \%$ do total. O IVI relativo ao grupo das cinco espécies mais representativas foi de 54,13\% (Tabela 6).

Ouratea hexasperma (Ochnaceae) foi a espécie mais destacada na comunidade arbórea, alcançando IVI de 19,24 \% do total. As demais espécies com elevado valor de importância fitossociológica foram: Caraipa savannarum (Clusiaceae), IVI = $12,76 \%$; Pouteria sp. 1 (Sapotaceae), IVI = 8,16\%; Eugenia sp. (Myrtaceae), IVI = 7,42\% e Vochysia rufa (Vochysiaceae), IVI = $6,55 \%$ (Tabela 6).

\section{ÁREA 4: CERRADO SENSU STRICTO}

Este cerrado apresentou maior riqueza e densidade de indivíduos do que as demais áreas, o que produziu menores valores relativos dos parâmetros fitossociológicos. No grupo das cinco espécies mais representativas, a abundância, a dominância e a freqüência relativa totalizaram $52,34 \%$ (correspondente a 948 indivíduos/ha), 41,31\% (Área Basal $=2,74 \mathrm{~m}^{2} \cdot \mathrm{ha}^{-1}$ ) e $39,88 \%$, respectivamente. O IVI calculado para essas espécies somou 44,51 \% do total do IVI (Tabela 6).

Novamente, Ouratea hexasperma foi a espécie com maior expressão fitossociológica, com IVI de $15,63 \%$ do total, seguida por Pouteria sp.3 (Sapotaceae), IVI = 8,48 \%; Myrtaceae sp.3, IVI $=7,72 \%$, Miconia sp. 1 (Melastomataceae), IVI $=6,56 \% \mathrm{e}$ Vochysia rufa (Vochysiaceae), IVI = 6,12 \% (Tabela 6).

\section{DISCUSSÃO}

Os cerrados estudados em Rondônia apresentaram riqueza de espécies, densidade e área basal comparáveis aos cerrados do
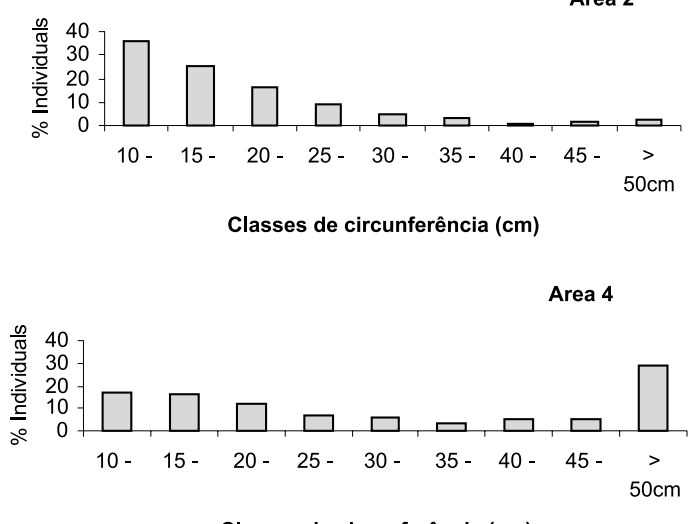

Classes de circunferência $(\mathrm{cm})$

Figura 1 - Distribuição de circunferência das árvores em quatro comunidades de cerrado de Vilhena, Rondônia. 
Tabela 6 - Parâmetros fitossociológicos de quatro áreas de cerrado inventariados no município de Vilhena, Rondônia. Destaque para as cinco espécies com maior IVI (Índice de Valor de Importância Específica, em \%). Legenda: N (Abundância Absoluta.ha-1), AB (Área Basal, em m².ha-1), NR (Abundância Relativa, em \%), DoR (Dominância Relativa, em \%), FR (Freqüência Relativa, em \%).

\begin{tabular}{|c|c|c|c|c|c|c|}
\hline ESPÉCIES & $\mathbf{N}$ & $A B\left(m^{2}\right)$ & $\%$ NR & $\%$ DoR & $\%$ FR & $\%$ IVI \\
\hline \multicolumn{7}{|l|}{$\begin{array}{c}\text { Área } 1 \\
\text { Cerradão }\end{array}$} \\
\hline Qualea multiflora & 166 & 1,48 & 10,32 & 11,90 & 8,94 & 10,39 \\
\hline $\begin{array}{l}\text { Byrsonima } \\
\text { crassifólia }\end{array}$ & 91 & 1,12 & 5,66 & 9,03 & 5,14 & 6,61 \\
\hline $\begin{array}{l}\text { Kielmeyera } \\
\text { rubriflora }\end{array}$ & 117 & 0,34 & 7,27 & 2,71 & 6,12 & 5,37 \\
\hline $\begin{array}{l}\text { Byrsonima } \\
\text { tomentosa }\end{array}$ & 64 & 0,63 & 3,98 & 5,10 & 3,79 & 4,29 \\
\hline Sclerolobium sp. & 47 & 0,94 & 2,92 & 7,60 & 2,20 & 4,24 \\
\hline $\begin{array}{c}\text { Total } \\
5 \text { espécies }>\text { IVI }\end{array}$ & 485 & 4,51 & 30,15 & 36,34 & 26,19 & 30,89 \\
\hline $\begin{array}{l}\text { Total demais } 55 \\
\text { espécies }\end{array}$ & 913 & 6,59 & 56,74 & 52,93 & 64,75 & 58,14 \\
\hline $\begin{array}{l}\text { Total troncos } \\
\text { mortos }\end{array}$ & 211 & 1,34 & 13,11 & 10,73 & 9,06 & 10,97 \\
\hline TOTAL GERAL & 1.609 & 12,44 & 100,00 & 100,00 & 100,00 & 100,00 \\
\hline \multicolumn{7}{|l|}{$\begin{array}{c}\text { Área } 2 \\
\text { Campo Sujo }\end{array}$} \\
\hline Eugenia sp. & 102 & 0,39 & 23,07 & 22,86 & 17,49 & 21,14 \\
\hline Miconia sp.1 & 64 & 0,26 & 14,48 & 14,96 & 15,59 & 15,01 \\
\hline Pouteria sp.1 & 46 & 0,18 & 10,41 & 10,58 & 11,41 & 10,80 \\
\hline $\begin{array}{l}\text { Connarus } \\
\text { suberosus }\end{array}$ & 41 & 0,11 & 9,28 & 6,39 & 9,88 & 8,52 \\
\hline Eriotheca sp.1 & 30 & 0,09 & 6,79 & 5,37 & 4,94 & 5,70 \\
\hline $\begin{array}{c}\text { Total } 5 \text { espécies } \\
>\text { IVI }\end{array}$ & 283 & 1,04 & 64,03 & 60,16 & 59,31 & 61,17 \\
\hline $\begin{array}{l}\text { Total demais } 21 \\
\text { espécies }\end{array}$ & 87 & 0,37 & 19,68 & 21,27 & 27,38 & 22,78 \\
\hline $\begin{array}{l}\text { Total troncos } \\
\text { mortos }\end{array}$ & 72 & 0,32 & 16,29 & 18,57 & 13,31 & 16,06 \\
\hline TOTAL GERAL & 442 & 1,72 & 100,00 & 100,00 & 100,00 & 100,00 \\
\hline \multicolumn{7}{|l|}{$\begin{array}{c}\text { Área } 3 \\
\text { Cerrado Sensu } \\
\text { Stricto } \\
\end{array}$} \\
\hline $\begin{array}{c}\text { Ouratea } \\
\text { hexasperma }\end{array}$ & 383 & 1,43 & 28,31 & 16,42 & 13,00 & 19,24 \\
\hline $\begin{array}{c}\text { Caraipa } \\
\text { savannarum }\end{array}$ & 127 & 2,05 & 9,39 & 23,58 & 5,30 & 12,76 \\
\hline Pouteria sp.1 & 106 & 0,65 & 7,83 & 7,45 & 9,21 & 8,16 \\
\hline Eugenia sp1 & 123 & 0,48 & 9,09 & 5,48 & 7,70 & 7,42 \\
\hline Vochysia rufa & 86 & 0,58 & 6,36 & 6,63 & 6,65 & 6,55 \\
\hline $\begin{array}{c}\text { Total } 5 \text { espécies } \\
>\text { IVI }\end{array}$ & 825 & 5,19 & 60,98 & 59,56 & 41,86 & 54,13 \\
\hline $\begin{array}{l}\text { Total demais } 34 \\
\text { espécies }\end{array}$ & 468 & 3,17 & 34,5898 & 36,42 & 52,53 & 41,18 \\
\hline $\begin{array}{l}\text { Total troncos } \\
\text { mortos }\end{array}$ & 60 & 0,35 & 4,43 & 4,02 & 5,61 & 4,69 \\
\hline TOTAL GERAL & 1.353 & 8,71 & 100,00 & 100,00 & 100,00 & 100,00 \\
\hline
\end{tabular}

Tabela 6 - continuação.

\begin{tabular}{|c|c|c|c|c|c|c|}
\hline ESPÉCIES & N & $A B\left(m^{2}\right)$ & $\%$ NR & $\%$ DoR & $\%$ FR & $\%$ IVI \\
\hline $\begin{array}{c}\text { Área } 4 \\
\text { Cerrado Sensu } \\
\text { Stricto }\end{array}$ & & & & & & \\
\hline $\begin{array}{c}\text { Ouratea } \\
\text { hexasperma }\end{array}$ & 428 & 0,83 & 23,63 & 12,54 & 10,72 & 15,63 \\
\hline Pouteria sp.3 & 149 & 0,65 & 8,23 & 9,74 & 7,47 & 8,48 \\
\hline Myrtaceae 3 & 160 & 0,37 & 8,83 & 5,65 & 8,67 & 7,72 \\
\hline Miconia sp.1 & 127 & 0,37 & 7,01 & 5,56 & 7,11 & 6,56 \\
\hline Vochysia rufa & 84 & 0,52 & 4,64 & 7,82 & 5,91 & 6,12 \\
\hline $\begin{array}{c}\text { Total } 5 \text { espécies } \\
>\text { IVI }\end{array}$ & 948 & 2,74 & 52,34 & 41,31 & 39,88 & 44,51 \\
\hline $\begin{array}{l}\text { Total demais } 40 \\
\text { espécies }\end{array}$ & 819 & 3,70 & 45,23 & 55,85 & 56,23 & 52,44 \\
\hline $\begin{array}{l}\text { Total troncos } \\
\text { Mortos }\end{array}$ & 44 & 0,19 & 2,43 & 2,84 & 3,89 & 3,05 \\
\hline
\end{tabular}

Brasil Central (Goodland, 1971, 1979; Ribeiro et al., 1985; Silberbauer-Gottsberger \& Eiten, 1987; Nascimento \& Saddi , 1992; Felfili \& Silva Junior, 1993).

O número de espécies encontrado nos cerrados amazônicos é geralmente baixo (Miranda et al., 2003; Sanaiotti et al., 1999). Os cerrados de Rondônia localizam-se na Amazônia, mas eles são unidos por corredores contínuos ao cerrado do Brasil Central. Portanto, devem ser considerados como o limite norte do domínio dos cerrados brasileiros e, embora eles localizem-se próximos das áreas isoladas dos cerrados amazônicos, são mais ricos que essas áreas isoladas.

Embora não tenham sido identificadas em nível específico, a maioria das espécies arbóreas encontradas nos cerrados estudados em Rondônia é comum às espécies que ocorrem nas principais áreas dos cerrados brasileiros, com exceção interessante de Caraipa savannarum, que não ocorre no Brasil Central (Mendonça et al., 1998). Sua distribuição vai das savanas do norte de América do Sul até os cerrados do sul da Amazônia brasileira, ocorrendo em grande abundância nas savanas das Guianas (Kubitzki, 1978). No Brasil, este é o primeiro registro de C. savannarum como espécie importante em comunidade arbóreas dos cerrados (segunda espécie mais importante da área 3), sugerindo que os cerrados de Rondônia possuem fortes relaçôes florísticas com o cerrados do Brasil Central, como também nas savanas do Norte de América do Sul, fato que demonstra sua característica de transição entre esses dois importantes domínios vegetacionais.

Quinze espécies apresentam ampla distribuição nos cerrados brasileiros: Qualea grandiflora, Qualea multiflora, Qualea parviflora, Kielmeyera coriacea, Lafoensia pacari, Roupala montana, Tabebuia ochracea, Tocoyena formosa, Xylopia aromatica, Bowdichia virgilioides, Byrsonima coccolobifolia, Caryocar brasiliensis, Connarus suberosus, Curatella americana e Hancornia speciosa (Ratter e Dargie, 1992; Ratter et al., 1996). 
Todos os parâmetros florísticos e estruturais analisados foram maiores na área 1 (cerradão-cerrado sensu stricto) e menores na área 2 (campo sujo), com exceção da porcentagem de árvores mortas. As diferenças florísticas e estruturais entre as áreas, assim como as diferenças entre os IVI de uma mesma espécie encontrada nas quatro áreas, podem estar relacionados a fatores edáficos. Goodland \& Pollard (1973) encontraram diferenças na flora, fisionomia e produtividade dos cerrados, associadas a variações edáficas.

Algumas espécies são importantes em algumas áreas e raras em outras, fato que pode indicar preferências pelas condições ecológicas de alguns ambientes. Por exemplo, Q. multiflora, a primeira em importância na área 1 , não ocorre nas outras áreas. a primeira em importância nas áreas 3 e 4, O. Hexasperma, não ocorre na área 1. Eugenia sp., a primeira em importância na área 2, foi também importante na área 3, mas rara nas áreas 1 e 4.

O fato de mais da metade das espécies terem sido registradas somente em uma das quatro áreas inventariadas, pode ser resultado da subamostragem dos ambientes, mas também, a evidência as limitações físicas e do grau de intervenção humana nessas áreas, uma vez que algumas espécies podem tolerar mais, tanto as variaçóes naturais dos ambientes, como as mudanças nos diversos fatores históricos e humanos como: freqüência de fogo, fragmentação e impactos resultantes dos diversos usos da terra. No entanto, esse resultado sugere que a estratégia para priorização de áreas para conservação deve levar em consideração a inclusão de partes representativas do mosaico florístico e fito-fisionômico dos cerrados.

Entre as espécies presentes em todas as áreas, algumas aparecem em elevadas densidades ou abundância, como exemplo Vochysia rufa, Qualea multiflora, Caryocar brasiliense e Bowdichia virgilioides. Isso sugere que um grupo de espécies está mais apto a colonizar diferentes ambientes em densidades elevadas. Entretanto, existem outras espécies que aparecem em uma única área, em densidades também elevadas como Pouteria sp.2, Caraipa savannarum e Eugenia sp.

As alturas das árvores nas áreas estudadas em Rondônia foram menores do que outras áreas dos cerrados do Brasil Central, mas são comparáveis a outros cerrados amazônicos. Goodland (1971) verificou altura média de três metros nos campos sujos, seis metros nos cerrados sensu stricto e nove metros no cerradão. SilberbauerGottsberger \& Eiten (1987) verificaram altura média de três metros em um cerrado de Botucatu, São Paulo. Nos cerrados amazônicos a altura encontrada por Miranda \& Absy (2000) foi entre 2,4 e $3 \mathrm{~m}$ para as savanas de Roraima. Para as savanas do Marajó, Pará, Bastos (1984) verificou altura entre três e quatro metros. Para o cerrado de Alter-do-Chão, Pará, Miranda (1993) verificou uma altura média de $3,18 \mathrm{~m}$.

De acordo com Ratter (1986), as espécies mais altas no cerrado sensu stricto estão entre cinco e seis metros de altura. No cerradão estão entre dez e doze metros, mas podem ocorrer indivíduos maiores. No presente estudo, as espécies que apresentaram as maiores alturas foram Sclerolobium sp., com indivíduos entre dez e quinze metros; Qualea multiflora, Byrsonima crassifolia, Ilex sp., Pouteria sp. 1 e Oenocarpus distichus apresentaram indivíduos com mais de dez metros, na área 1.

As distribuiçōes de circunferência das quatro áreas revelaram, na maioria distribuiçôes decrescentes, muitos indivíduos nas classes de tamanhos menores, sendo o mesmo padrão de distribuição apresentado pela maioria das principais populações. De acordo com Richards (1996) esta distribuição é característica de vegetaçóes naturais onde um grande número de indivíduos pequenos predomina.

O decréscimo acentuado apresentado na primeira classe de tamanho, especialmente na área 3 e 4, pode ser devido a não ocorrência de fogo nos últimos dois anos, segundo informação dos moradores e observaçóes de campo. Esse fator pode ter proporcionado o estabelecimento de indivíduos da primeira classe de circunferência, entre dez e quinze centímetros, permitindo-os passar para as classes superiores. $\mathrm{O}$ fogo afeta classes de tamanho de um modo diferenciado (Raw \& Hay, 1985) e determina o estabelecimento de muitos arbustos e árvores (Hoffman, 1996). Mudanças na periodicidade dos eventos fenológicos também podem influenciar o número de indivíduos das primeiras classes de tamanho (Miranda, 1991).

A porcentagem de árvores mortas foi maior na área 2, no entanto, foi também alta na área 1 . Segundo informação dos moradores e observações de campo, essas áreas foram queimadas na última estação seca e, a mortalidade alta pode ser devida à incidência de fogo recente.

A percentagem de árvores mortas nas áreas 1 e 2 são comparáveis àquelas encontradas no cerrado do Parque Nacional de Brasília (13,71\% de troncos mortos), que também havia sido queimado antes da pesquisa (Felfili \& Silva Junior, 1993). As porcentagens de árvores mortas nas áreas 3 e 4 foram muito menores doa que as encontradas nas áreas protegidas dos cerrados de Brasília, como por exemplo, na Estação Ecológica de Águas Emendadas (5,11\% de troncos mortos) e na Área de Proteção Ambiental Cabeça de Veado (5,67\% de troncos mortos) e dos cerrados de Minas Gerais estudados nos municípios de Patrocínio (5,20\% de troncos mortos) e Paracatu (6,02\% de troncos mortos) (Felfili \& Silva Junior, 1993).

As manchas de cerrado apresentaram consideráveis níveis de mortalidade, provavelmente associados ao fogo, especialmente, elevados no cerradão e no campo sujo. Em geral, áreas com menor índice de lenhosidade, como campo sujo ou campo cerrado, podem apresentar maior vulnerabilidade ao fogo, embora a mortalidade associada possa variar de um estrato a outro (Raw $\&$ Hay, 1985). Assim, em campos sujos, a adaptação direcionada a tolerar o fogo pode atuar mais fortemente em ervas, enquanto que em cerrados sensu stricto, a seleção pode favorecer o estrato 
arbóreo que, por conseqüência, apresente menores taxas de mortalidade associadas a esse evento, conforme evidenciado neste estudo. No entanto, não se deve desconsiderar fatores históricos e antrópicos para explicar parte da mortalidade observada.

\section{CONCLUSÕES}

Os cerrados de Rondônia estudados apresentaram composição florística e estrutura de tamanho diferenciadas, evidenciando o mosaico florístico e fisionômico que caracteriza esse bioma.

Em relação ao componente arbóreo, o cerradão foi o ambiente com maior riqueza de espécies e maior porte, enquanto que o campo sujo evidenciou menor diversidade e porte.

O componente arbóreo é dominado por um grupo restrito de espécies, com exceção do cerradão onde a riqueza de espécies parece diluir a dominância entre um número maior de táxons.

Os cerrados de Rondônia têm afinidade florística e fisionômica com os existentes no Brasil Central, podendo ser considerados como uma extensão transicional desse bioma para o domínio da floresta Amazônia.

A mortalidade de árvores associada ao fogo varia entre as áreas estudadas, mas as espécies do cerrado sensu stricto parecem suportar melhores os efeitos deste fenômeno.

\section{AGRADECIMENTOS}

Este trabalho foi financiado com recursos do Programa PLANAFLORO, através da Empresa TECNOSOLO. Nós agradecemos a Edgar Menezes e Donadoni, pelo apoio logístico; aos fazendeiros Joaquim Romeu Fontes, José Paulo Gomes, Mário Alexandre Kerber, Romeu Kerber, que permitiram o trabalho em suas propriedades; ao Marcelo Cordeiro Thales pela produção do mapa de localização das áreas estudadas e aos botânicos que ajudaram na identificação das espécies: Kikyo Yamamoto, Volker Bittrich, Jorge Tamashiro (UNICAMP) e José Pirani (USP).

\section{BIBLIOGRAFIA CITADA}

Bastos, M.N.C. 1984. Levantamento Florístico do Estado do Pará. I- Campos de Joanes (Ilha de Marajó). Boletim do MPEG, série Botânica, 1: 67-86.

Borhidi, A. 1988. Vegetation dynamics of the savannization process on Cuba. Vegetatio, 77: 177-183.

Brower, J.E; Zar, J.H.; von Ende, C.N. 1997. Field and laboratory methods for general Ecology. $4^{\text {th }}$ ed. WCB McGraw-Hill, New York. 273pp.

Coutinho, L.M. 1978. O conceito do Cerrado. Revista Brasileira de Botânica, 1: 17-23.

Durigan, G.; Leitão Filho, H.F.; Rodrigues, R.R. 1994. Phytosociology and structure of a frequently burnt cerrado vegetation in SE-Brazil. Flora, 189: 153-160.

Eiten, G. 1972. The cerrado vegetation of Brazil. The Botanical
Review, 38: 201-341.

Felfili, J.M.; Silva Junior, M.C. 1993. A comparative study of cerrado (Sensu stricto) vegetation in Central Brazil. Journal of Tropical Ecology, 9: 277-289.

Furley, P.A.; Ratter, J.A. 1988. Soil resouces and communities of the Central Brazilian cerrado and their development. Journal of Biogeography, 15: 97-108.

Gibbs, P.E., Leitão Filho, H.F.; Shepherd, G.J. 1983. Floristic Composition and community structure in an area of cerrado in SE Brazil. Flora, 173: 433-449.

Goodland, R. 1971. A physionomic analysis of the cerrado vegetation of Central Brazil. Journal of Ecology, 59: 411-419.

Goodland, R. 1979. Análise ecológica da vegetação do cerrado. In: Goodland, R.J.A.; Ferri, M.G. (Eds.) Ecologia do Cerrado. EDUSP, São Paulo. p.61-160.

Goodland, R.; Pollard, R. 1973. The brazilian cerrado vegetation: a fertility gradient. Journal of Ecology, 61: 219-224.

Hoffmann, W.A. 1996. The effects of fire and cover on seedling establishment in a neotropical savanna. Journal of Ecology, 84: 383-393.

Huber, O. 1982. Significance of savanna vegetation in the Amazon Territory of Venezuela. In: Prance, G.T. (Ed.) Biological Diversification in the Tropics. Columbia University Press, New York. p. 221-244.

Huber, O. 1987. Neotropical Savannas: Their Flora and Vegetation. Tree, 2: 67-71.

Kubitzki, K. 1978. Caraipa and Mahurea (Bonnetiaceae). Memoirs of New York Botanical Garden, 29: 82-138.

Magurran, A. E. 2004. Measuring Biological Diversity. Blackwell Publishing, Malden. 256p.

Medina, E. 1987. Nutrients: requirements, conservation and cycles in the herbaceous layer. In: Walker, B.W. (ed.) Determinants of savannas. IRL Press, Oxford (IUBS monograph series no. 3). p.39-67.

Mendonça, R.C., Felfili, J.M., Walter, B.M., Silva Jr, M.C., Rezende, A.V., Filgueiras, T.S.; Nogueira, P.E. 1998. Flora vascular do Cerrado. In: Sano, S.M.; Almeida, S.P. (Eds.) Cerrado - Ambiente e Flora. EMBRAPA-CPAC, Planaltina. p.289-539.

Miranda, I.S. 1991. Estrutura e Fenologia da comunidade arbórea do cerrado amazônico de Alter-do-chão, Pará. Dissertação de Mestrado, Instituto Nacional de Pesquisas da Amazônial Fundação Universidade do Amazonas, Manaus, Amazonas. 198 pp.

Miranda, I.S. 1993. Estrutura do estrato arbóreo do cerrado amazônico em Alter-do-Chão, Pará, Brasil. Revta. brasil. Bot., 16: 143-150.

Miranda, I.S.; Absy, M.L. 2000. Fisionomia das Savanas de Roraima. Acta Amazonica, 30: 423-440.

Miranda, I.S.; Absy, M.L.; Rebêlo, G.H. 2003. Community Structure of Woody Plants of Roraima Savannahs, Brazil. Plant Ecology, 164: 109-123.

Montgomery, R.F.; Askew, G.P. 1983. Soils of tropical savannas. In: 


\section{ACTA

Bourliere, F. (Ed.) Tropical Savannas Ecosystems of the World. Elsevier, Amsterdam. p.63-78.

Nascimento, M.T.; Saddi, N. 1992. Structure and floristic composition in an area of cerrado in Cuiabá-MT, Brazil. Revta brasil. Bot., 15: 47-56.

Nimer, E. 1991. Clima. In: IBGE, Geografia do Brasil: Região Norte, Vol. 3. IBGE, Rio de Janeiro. p.61-71.

Oliveira Filho, A.T.; Martins, F.R. 1986. Distribuição, caracterização e composição florística das formaçōes vegetais da Salgadeira, na Chapada dos Guimarães (MT). Revta brasil. Bot., 9: 207-223.

Oliveira Filho, A.T., Shepherd, J., Martins, F.R.; Stubblebine, W.H. 1989. Environmental factors affecting physiognomic and floristic variation in an area cerrado in central Brazil. Journal of Tropical Ecology, 5: 413-431.

Pagano, S.N., Cesar, O.; Leitão Filho, H.F. 1989. Estrutura Fitossociológica do Estrato Arbustivo-Arbóreo da Vegetação de Cerrado da Área de Proteção Ambiental (APA) de Corumbataí - Estado de São Paulo. Rev. Brasil. Biol., 49: 49-59.

Ratter, J.A. 1986. Notas sobre a vegetação da Fazenda Água Limpa (Brasília, DF, Brazil). Editora UnB, Brasília (Textos Universitários $\mathrm{n}^{\circ} .3$ ). 133pp.

Ratter, J. A.; Dargie, T.C.D. 1992. An analysis of the floristic composition of 26 cerrado areas in Brazil. Edin. J. Bot., 49: 235250.

Ratter, J.A.; Bridgewater, S., Atkinson, R.; Ribeiro, J.F. 1996. Analisys of the Floristic Composition of the Brazilian Vegetation II: Comparison of the Woody Vegetation of 98 Areas. Edinb. J. Bot., 53: 152-180.
Raw, A.; Hay, J. D. 1985. Fire and other factors affecting a population of Simaruba amara in a "cerradão" near Brasília, Brasil. Revta. Brasil. Bot., 9: 101 - 107.

Ribeiro, J.F., Silva, J.C.S.; Batmanian, G.J. 1985. Fitossociologia de tipos fisionômicos de cerrado de Planaltina, DF. Revta. Brasil. Bot., 8: 131-142.

Ribeiro, J.F.; Walter, B.M.T. 1998. Fitofisionomias do Bioma Cerrado. In: Sano, S.M.; Almeida, S.P. (Eds.) Cerrado - Ambiente e Flora. EMBRAPA-CPAC, Planaltina. p.89-168.

Richards, P.W. 1996. The Tropical Rain Rorest: an ecological study ( $2^{\text {nd }}$. Ed.). Cambridge University Press, Cambridge. 573p.

Sanaiotti, T.M.; Bridgewater, S.; Ratter, J.A. 1999. A floristic study of the savanna vegetation of the state of amapá, Brazil, and suggestions for its conservation. Bol. Mus. Para. Emílio Goeldi, série Botânica, 13(1): 3-29.

Silberbauer-Gottsberger, I.; Eiten, G. 1987. A Hectare of Cerrado. I. General Aspects of the Trees and Thick-Stemmed Shrubs. Phyton, 27: 55-91.

Recebido em 04/05/2006

Aceito em 03/11/2006 
Anexo I - Listagem geral das espécies de árvores registradas em quatro áreas de cerrado do município de Vilhena, Rondônia. Legenda Área: Área 1 - Cerradão, Área 2 - Campo Sujo, Área 3 - Cerrado Sensu Stricto, Área 4 - Cerrado Sensu Stricto.

\begin{tabular}{|c|c|c|c|c|c|}
\hline \multirow{2}{*}{ №. Espécies } & \multicolumn{5}{|c|}{$\mathrm{N}^{0}$. Indivíduos.ha- ${ }^{-1}$} \\
\hline & \multirow{2}{*}{\multicolumn{2}{|c|}{ Área 1 Área 2}} & \multirow[t]{2}{*}{ Área 3} & \multirow[t]{2}{*}{ Área 4} & \multirow[t]{2}{*}{ Total } \\
\hline I. Annonaceae & & & & & \\
\hline 1 Annona coriacea Mart. & 5 & 10 & 6 & 9 & 30 \\
\hline 2 Guateria sp. & 4 & & & & 4 \\
\hline 3 Xylopia aromatica (Lam.) Mart. & & & & 3 & 3 \\
\hline \multicolumn{6}{|l|}{ II. Apocynaceae } \\
\hline 4 Aspidosperma sp. & 6 & & & & 6 \\
\hline 5 Hancornia speciosa Gomes & 1 & & 86 & 35 & 122 \\
\hline $\begin{array}{l}\text { Himatanthus sucuuba (Spr. ex Müll. } \\
\text { Arg.)Wood. }\end{array}$ & 4 & & & 5 & 9 \\
\hline \multicolumn{6}{|l|}{ III. Aquifoliaceae } \\
\hline 7 Ilex sp. & 20 & 1 & 4 & 2 & 27 \\
\hline \multicolumn{6}{|l|}{ IV. Arecaceae } \\
\hline 8 Bactris sp. & 29 & & & & 29 \\
\hline 9 Mauritiella sp. & 5 & & & & 5 \\
\hline 10 Oenocarpus distichus Mart. & 4 & & & & 4 \\
\hline 11 Arecaceae 2 & 5 & 46 & 106 & & 157 \\
\hline \multicolumn{6}{|l|}{ V. Asteraceae } \\
\hline 12 Eremanthus sp. & 3 & & 9 & 105 & 117 \\
\hline 13 Vernonia sp. & 1 & & & & 1 \\
\hline \multicolumn{6}{|l|}{ VI. Bignoniaceae } \\
\hline 14 Tabebuia ochracea (Cham.) Standl. & 54 & & & 3 & 57 \\
\hline \multicolumn{6}{|l|}{ VII. Bombacaceae } \\
\hline 15 Eriotheca sp.1 & 3 & 30 & & & 33 \\
\hline 16 Eriotheca sp.2 & 5 & & & 1 & 6 \\
\hline 17 Eriotheca sp.3 & 29 & & & & 29 \\
\hline 18 Pseudobombax sp. & & & 1 & 13 & 14 \\
\hline \multicolumn{6}{|l|}{ VIII. Burseraceae } \\
\hline 19 Protium sp. & 49 & & & & 49 \\
\hline \multicolumn{6}{|l|}{ IX. Caesalpiniaceae } \\
\hline 20 Bauhinia sp. & 3 & & & & 3 \\
\hline 21 Sclerolobium sp. & 51 & & & & 51 \\
\hline \multicolumn{6}{|l|}{ X. Caryocaraceae } \\
\hline 22 Caryocar brasiliense Cambess. & 7 & 3 & 47 & 23 & 80 \\
\hline \multicolumn{6}{|l|}{$\mathrm{XI}$. Celastraceae } \\
\hline 23 Maytenus sp. & 1 & & & & 1 \\
\hline \multicolumn{6}{|l|}{ XII. Chrysobalanaceae } \\
\hline 24 Couepia sp. & & & 1 & & 1 \\
\hline 25 Licania humilis Cham. \& Schltdl. & & 1 & 27 & 27 & 55 \\
\hline \multicolumn{6}{|l|}{ XIII. Clusiaceae } \\
\hline 26 Caraipa savannarum Kubitzki & & & 127 & & 127 \\
\hline 27 Kielmeyera coriacea Mart. \& Zucc. & 53 & 1 & & & 54 \\
\hline 28 Kielmeyera rubriflora Cambess. & 117 & & 7 & 6 & 130 \\
\hline
\end{tabular}

Anexo I - Continuação

\begin{tabular}{|c|c|c|c|c|c|}
\hline \multirow{2}{*}{ №. Espécies } & \multicolumn{5}{|c|}{ №. Indivíduos. ha-1 } \\
\hline & Área 1 & Área 2 & Área 3 & Área 4 & Total \\
\hline \multicolumn{6}{|l|}{ XIV. Combretaceae } \\
\hline 29 Combretaceae 1 & & & & 16 & 16 \\
\hline 30 Combretaceae 2 & & & 8 & 3 & 11 \\
\hline \multicolumn{6}{|l|}{ XV. Connaraceae } \\
\hline 31 Connarus suberosus Planch. & & 41 & 21 & 37 & 99 \\
\hline 32 Curatella americana L. & 26 & & & & 26 \\
\hline 33 Davilla sp.1 & 47 & & 4 & 5 & 56 \\
\hline 34 Davilla sp.2 & 15 & 1 & & & 16 \\
\hline \multicolumn{6}{|l|}{ XVI. Erythoxylaceae } \\
\hline 35 Erythroxylum sp.1 & 22 & 3 & 27 & 7 & 59 \\
\hline 36 Erythroxylum sp.2 & 3 & & 1 & 9 & 13 \\
\hline \multicolumn{6}{|l|}{ XVII. Euphorbiaceae } \\
\hline 37 Maprounea guianensis Aubl. & & & & 2 & 2 \\
\hline \multicolumn{6}{|l|}{ XVIII. Fabaceae } \\
\hline 38 Andira sp. & & 4 & 4 & & 8 \\
\hline 39 Bowdichia virgilioides Kunth. & 50 & 1 & 18 & 7 & 76 \\
\hline 40 Dalbergia sp. & 12 & & & & 12 \\
\hline 41 Pterodon cf. emarginatus Vog. & 13 & & & 18 & 31 \\
\hline 42 Vatairea sp. & 3 & & & & 3 \\
\hline \multicolumn{6}{|l|}{ XIX. Flacourtiaceae } \\
\hline 43 Casearia sp. & 2 & & & & 2 \\
\hline \multicolumn{6}{|l|}{ XX. Humiriaceae } \\
\hline 44 Humiriaceae Indet. & & & 7 & 20 & 27 \\
\hline \multicolumn{6}{|l|}{ XXI. Icacinaceae } \\
\hline 45 Emotum nitens & 2 & & & 3 & 5 \\
\hline \multicolumn{6}{|l|}{ XXII. Indeterminada } \\
\hline 46 Indeterminada 1362 & 7 & & & & 7 \\
\hline \multicolumn{6}{|l|}{ XXII. Lauraceae } \\
\hline 47 Lauraceae Indet. & & & & 21 & 21 \\
\hline \multicolumn{6}{|l|}{ XXIII. Lecythidaceae } \\
\hline 48 Eschweilera nana (0. Berg) Miers & & 3 & 20 & 14 & 37 \\
\hline \multicolumn{6}{|l|}{ XXIV. Lythraceae } \\
\hline 49 Lafoensia pacari A. St.-Hil. & 18 & & & & 18 \\
\hline \multicolumn{6}{|l|}{ XXV. Malpighiaceae } \\
\hline 50 Byrsonima cf. intermedia A. Juss. & & 4 & & & 4 \\
\hline 51 Byrsonima coccolobaefolia Kunth & 10 & 1 & 21 & 18 & 50 \\
\hline 52 Byrsonima crassifolia Nied. & 91 & & 9 & 22 & 122 \\
\hline 53 Byrsonima sp. & 21 & & 9 & & 30 \\
\hline 54 Byrsonima sp.1 & & & & 31 & 31 \\
\hline 55 Byrsonima tomentosa & 64 & & & & 64 \\
\hline \multicolumn{6}{|l|}{ XXVI. Melastomataceae } \\
\hline 56 Miconia albicans (Sw.) Triana & 19 & & & & 19 \\
\hline 57 Miconia sp.1 & & 64 & 55 & 127 & 246 \\
\hline 58 Miconia sp.2 & & 15 & 6 & 54 & 75 \\
\hline 59 Miconia stenostachya DC. & 7 & & & & 7 \\
\hline
\end{tabular}


Anexo I - Continuação

\begin{tabular}{|c|c|c|c|c|c|}
\hline \multirow{2}{*}{ №. Espécies } & \multicolumn{5}{|c|}{$\mathrm{N}^{0}$. Indivíduos.ha- ${ }^{-1}$} \\
\hline & Área 1 & 1 Área 2 & Área 3 & Área 4 & Total \\
\hline \multicolumn{6}{|l|}{ XXVII. Memecylaceae } \\
\hline 60 Mouriri elliptica Mart. & & 6 & 21 & 37 & 64 \\
\hline \multicolumn{6}{|l|}{ XXVIII. Mimosaceae } \\
\hline 61 Enterolobium cf. ellipticum Benth. & & & 1 & & 1 \\
\hline 62 Stryphnodendron sp. & & & & 5 & 5 \\
\hline \multicolumn{6}{|l|}{ XXIX. Myristicaceae } \\
\hline 63 Virola sebifera Aubl. & & 4 & 1 & & 5 \\
\hline \multicolumn{6}{|l|}{ XXX. Myrtaceae } \\
\hline 64 Eugenia aurata 0 . Berg & 10 & & & & 10 \\
\hline 65 Eugenia sp.2 & 13 & 102 & & 23 & 138 \\
\hline 66 Eugenia sp1 & & & 123 & & 123 \\
\hline 67 Marlierea sp. & & & 5 & & 5 \\
\hline 68 Myrtaceae 1 & & & & 3 & 3 \\
\hline 69 Myrtaceae 2 & 2 & & & & 2 \\
\hline 70 Myrtaceae 3 & & & 5 & 160 & 165 \\
\hline \multicolumn{6}{|l|}{ XXXI. Nyctaginaceae } \\
\hline 71 Neea sp. & 9 & & & & 9 \\
\hline \multicolumn{6}{|l|}{ XXXII. Ochnaceae } \\
\hline 72 Ouratea hexasperma (A. St.-Hil.) Baill. & & 7 & 383 & 428 & 818 \\
\hline \multicolumn{6}{|l|}{ XXXIII. Proteaceae } \\
\hline 73 Roupala Montana Aubl. & 9 & & & & 9 \\
\hline \multicolumn{6}{|l|}{ XXXIV. Rubiaceae } \\
\hline 74 Alibertia sp. & 22 & & & & 22 \\
\hline 75 Ferdinandusa elliptica Pohl & & & 4 & & 4 \\
\hline 76 Guettarda sp. & 12 & & & & 12 \\
\hline 77 Palicourea rígida Kunth & 4 & & & & 4 \\
\hline 78 Rubiaceae Indet 1 & 3 & & & & 3 \\
\hline 79 Rubiaceae Indet.2 & 47 & 1 & 2 & 5 & 55 \\
\hline $\begin{array}{l}80 \text { Tocoyena formosa (Cham.\& Schltdl.) } \\
\text { K.Schum. }\end{array}$ & 34 & & & & 34 \\
\hline \multicolumn{6}{|l|}{ XXXV. Sapotaceae } \\
\hline 81 Pouteria sp.1 & 2 & & & & 2 \\
\hline 82 Pouteria sp.2 & & & & 149 & 149 \\
\hline 83 Pouteria sp.3 & & 8 & 16 & & 24 \\
\hline \multicolumn{6}{|l|}{ XXXVI. Scrophulariaceae } \\
\hline 84 Esterhazia macrodonta Bartl. & & & & 1 & 1 \\
\hline \multicolumn{6}{|l|}{ XXXVI. Simaroubaceae } \\
\hline 85 Simarouba amara Aubl. & 22 & & 9 & 1 & 32 \\
\hline \multicolumn{6}{|l|}{ XXXVII. Styracaceae } \\
\hline 86 Styrax camporum Pohl & & 2 & & 11 & 13 \\
\hline \multicolumn{6}{|l|}{ XXXVIII. Vochysiaceae } \\
\hline 87 Qualea grandiflora Mart. & 166 & & & & 166 \\
\hline 88 Qualea multiflora Mart. & 10 & 4 & 1 & 91 & 106 \\
\hline 89 Qualea parviflora Mart. & 80 & & & & 80 \\
\hline 90 Vochysia cinnamomea Pohl & & & & 25 & 25 \\
\hline
\end{tabular}

Anexo I - Continuação

\begin{tabular}{lccccc}
\hline \multirow{2}{*}{ No. Espécies } & \multicolumn{5}{c}{$\mathbf{N}^{0}$. Indivíduos.ha } \\
\cline { 2 - 6 } & Área 1 & Área 2 & Área 3 & Área 4 & Total \\
\hline 91 Vochysia haenkeana Mart. & 28 & & 86 & 84 & 198 \\
\hline 92 Vochysia rufa Mart. & 34 & 7 & 5 & 98 & 144 \\
\hline Total troncos vivos & $\mathbf{1 . 3 9 8}$ & $\mathbf{3 7 0}$ & $\mathbf{1 . 2 9 3}$ & $\mathbf{1 7 6 7}$ & $\mathbf{4 . 8 2 8}$ \\
\hline Total troncos mortos & $\mathbf{2 1 1}$ & $\mathbf{7 2}$ & $\mathbf{6 0}$ & $\mathbf{4 4}$ & $\mathbf{3 8 7}$ \\
\hline Total geral & $\mathbf{1 . 6 0 9}$ & $\mathbf{4 4 2}$ & $\mathbf{1 . 3 5 3}$ & $\mathbf{1 8 1 1}$ & $\mathbf{5 . 2 1 5}$ \\
\hline
\end{tabular}


